\title{
Configuração dos remanescentes florestais em uma área da Mata Atlântica do nordeste do Brasil: orientando medidas de conservação em escala municipal
}

\author{
Configuration of the forest remnants in an area of the Atlantic forest of northeast Brazil: guiding \\ conservation measures at the municipality level
}

\author{
E. R. Nascimento ${ }^{1 *}$; J. L. Santos ${ }^{2}$; S. F. Gouveia ${ }^{1}$ \\ ${ }^{1}$ Departamento de Ecologia/Laboratório de Biologia da Conservação, Universidade Federal de Sergipe, 49100-000, Aracaju- SE, \\ Brasil \\ ${ }^{2}$ Departamento de Ecologia/Laboratório de Entomologia, Universidade Federal de Sergipe, 49100-000, Aracaju - SE, Brasil \\ *erivelton.ufs@gmail.com
}

(Recebido em 29 de janeiro de 2016; aceito em 30 de março de 2016)

\begin{abstract}
A tomada de decisões para o manejo e conservação florestal em escalas locais (e.g., municípios) permite envolver a comunidade e atores diretamente ligados ao uso de recursos naturais. Quando informações detalhadas sobre alvos de conservação (e.g., espécies ameaçadas) são escassas, uma alternativa é utilizar parâmetros espaciais em escala da paisagem para orientar a tomada de decisão em escala local. Neste estudo, utilizamos o município de Umbaúba, localizado no sul do Estado de Sergipe, Brasil, um dos principais consumidores de produtos energéticos florestais do estado, para avaliar a configuração dos remanescentes florestais em escala da paisagem, a fim de contribuir para a tomada de decisões de conservação e manejo dos remanescentes no município. Utilizando um mapa dos fragmentos florestais do município (64,9 metros de resolução), analisamos um conjunto métricas de paisagem, incluindo quantidade de cobertura, forma e conectividade. Identificamos 35 (503 ha) fragmentos de três tipologias de florestas estacionais: arbustiva, arbustivo-arbórea e arbórea. Em geral, os remanescentes são pequenos, irregulares e com baixos índices de conectividade, típicos de áreas altamente fragmentadas. Apesar da baixa condição florestal, a classe arbórea teve a maior cobertura, incluindo o remanescente com maiores tamanho e conectividade (conhecido como Mata do Escôncio). Além de ser o maior fragmento de Umbaúba, ele se estende ao município de Indiaroba, formando um remanescente de maior tamanho (544 ha). Além disso, este remanescente abriga espécies ameaçadas de extinção como o macaco-guigó (Callicebus coimbrai) e a nascente do rio Indiaroba, com importância para a manutenção de serviços ecossistêmicos locais.

Palavras-chave: Mata Atlântica, ecologia de paisagem, tipologias florestais
\end{abstract}

Decision making for forest management and conservation on local scales (e.g., municipalities) allows the participation of the local community and the stakeholders involved in the use of natural resources. When detailed information on conservation targets (e.g., threatened species) are scarce, the use of spatial parameters on a landscape scale to guide local-scale decision making is an alternative. Here, we use the municipality of Umbaúba, located in the southern state of Sergipe, Brazil, and one of the main consumers of timber energy products in the state, to evaluate the configuration of the forest remnants on a landscape scale, in order to contribute to the decision making for local conservation and management of the forests in this municipality. Using a map of forest fragments (64.9 meters resolution), we analyzed a set landscape metrics, namely the amount of cover, shape and connectivity. We identified 35 fragments (503 hectares) of three different seasonal forest types: shrub, shrub and arboreal contact, and arboreal. In general, fragments are smallsized, irregularly shaped and show low connectivity indices, which is typical of highly fragmented areas. Despite the poor forest condition, the arboreal class had a larger cover, with the larger and more connected fragment, known as 'Escôncio' woods. Further than being the larger fragment in Umbaúba, it extends into the neighbor municipality, Indiaroba, comprising a larger remnant (544 ha). In addition, this remnant houses population of species threatened with extinction, such as the Coimbra-Filho's titi monkey (Callicebus coimbrai) and the river spring of the Indiaroba River, with significance for the maintenance of local ecosystem services.

Keywords: Atlantic Forest, Landscape metrics, Vegetation class 


\section{INTRODUÇÃ̃O}

Ações de manejo e conservação de recursos naturais requerem informações relevantes sobre os elementos a serem preservados, de maneira a suportar a tomada de decisão de medidas efetivas [1]. Geralmente, informações sobre a biodiversidade ou processos ecossistêmicos são capazes de subsidiar essa tomada de decisões para uma ampla escala de medidas de conservação ambiental, mas muitos desses dados são escassos para regiões ou localidades de interesse, sobretudo nas regiões tropicais [2]. Uma alternativa para orientar decisões de conservação e manejo é, portanto, utilizar indicadores ambientais que possam refletir padrões ou processos ecológicos de interesse. Em escala local, métricas da paisagem podem indicar diversos aspectos ecológicos de interesse, tais como diversidade de espécies, permeabilidade espacial para dispersão da fauna e flora, suscetibilidade a invasões biológicas, entre outros [3,4].

Neste contexto, o conhecimento dos padrões de cobertura florestal é fundamental nas ações de planejamento, conservação e recuperação ambiental de recursos florestais. Por exemplo, remanescentes maiores tendem a acomodar maiores populações de espécies e maior diversidade local; maior conectividade pode favorecer uma maior dispersão das espécies de uma localidade; e formas mais regulares podem abrigar espécies mais sensíveis a perturbações ambientais, devido a um menor efeito de borda [5,6]. Em contrapartida, a perda de habitat e da conexão entre fragmentos florestais pode provocar mudanças na estrutura e na dinâmica das populações, incluindo redução do potencial de dispersão e colonização das espécies, gerando uma diminuição do fluxo gênico das populações. [6,7,8].

No Brasil, o bioma mais afetado pela perda e fragmentação florestal é a Mata Atlântica [9]. A Mata Atlântica é formada por um conjunto de formações florestais, tais como Ombrófila Densa, Ombrófila Mista, Estacional Semidecidual, Estacional Decidual, Ombrófila Aberta e também se associa a ecossistemas como as restingas, manguezais e campos de altitude [10]. O bioma abrange tanto a região litorânea como os planaltos e serras, cobrindo do Rio Grande do Norte até o Rio Grande do Sul, ao longo de toda costa brasileira [11]. O bioma se enquadra em um dos 34 hotspots mundiais para a conservação da biodiversidade do planeta. [12]. Essas áreas formam um mosaico de biodiversidade, abrigando mais de $60 \%$ de todas as espécies terrestres do planeta [13]. Apesar de possuir uma grande biodiversidade, a Mata Atlântica é um dos ecossistemas mais afetados por ações antrópicas. Sua devastação teve início nas primeiras décadas de 1500 e o que restou da sua cobertura vegetal original foram fragmentos com diversos graus de isolamento e conectividade [14, 15]. Atualmente a Mata Atlântica brasileira possui cerca de 12,5\% do seu território original, rica em biodiversidade e alto endemismo [16]. Das 1.711 espécies de vertebrados, estima-se que cerca de 700 são endêmicas. [12].

No Estado de Sergipe de acordo com a lei n ${ }^{\circ} 11.428$ que dispõe sobre a utilização e proteção da vegetação nativa do bioma Mata Atlântica, a área original de mata no Estado era de 1018955 ha $(<1 \%$ da Mata Atlântica do Brasil), no entanto atualmente resta cerca de 7,1\% desse valor. Mesmo com a degradação de seu domínio o bioma ainda possui uma diversidade biológica considerável, abrigando espécies de elevado interesse conservacionista [17,18]. A região Sul do estado de Sergipe concentra os maiores remanescentes de Mata Atlântica, principalmente nos municípios de Estância, Santa Luzia do Itanhy, Umbaúba e Cristinápolis [19], embora não possua nenhuma unidade de conservação de proteção integral.

A cidade de Umbaúba, situada no litoral sul Sergipano, possui uma área correspondente a 11,88 mi ha, tendo um contingente populacional de 22.434 habitantes. O nome do município faz referência ao nome popular das espécies do gênero Cecropia [20]. O município é um dos principais consumidores de produtos energéticos florestais possuindo um consumo anual de 22.020,91 metros estéreos (st) de lenha e carvão mineral equivalente em lenha, usados para atender a demanda do setor industrial, de comércio e serviços, fato que tem contribuindo para os elevados índices de desmatamento da Mata Atlântica na região [19]. O município consome mais produtos florestais do que produz, tendo um saldo negativo desses produtos, necessitando importá-los de outros municípios para serem utilizados nas unidades de produção e em estabelecimentos de comércio e serviços [19]. A seleção do município para realização do estudo foi determinada aleatoriamente entre os municípios da região Sul do Estado de Sergipe, região em que se localizam os maiores remanescentes florestais do estado.

O trabalho foi realizado com o intuito de contribuir para o plano Municipal de Mata Atlântica, projeto da Fundação SOS Mata Atlântica, que têm por finalidade reunir e normatizar os elementos necessários à proteção, conservação, recuperação e uso sustentável da Mata Atlântica. Os planos materializarão as leis do bioma Mata Atlântica, a fim de proteger a floresta mais ameaçada do Brasil [16]. Nesse sentido o objetivo desse trabalho foi utilizar informações na escala da paisagem para avaliar a situação dos remanescentes 
florestais em Umbaúba a fim de contribuir com a tomada de decisões para a seleção de áreas prioritárias para conservação e recuperação ambiental do município.

\section{MATERIAL E MÉTODOS}

Utilizamos um mapeamento dos fragmentos florestais do município de Umbaúba em uma resolução espacial de 64,9 metros (Figura 1), obtido do banco de dados do Mapeamento Florestal de Sergipe de 2014 [19]. Essa classificação foi elaborada a partir da interpretação visual de imagens dos satélites CBERS e SPOT, com suporte de aerofotografias do litoral do estado, a qual resultou na identificação de 11 classes de uso e cobertura vegetal para o estado: Caatinga Arbustiva, Caatinga Arbustivo-Arbórea, Caatinga Arbórea, Contato (Regeneração), Contato Arbóreo, Floresta Estacional (Regeneração), Floresta Estacional Arbórea, Cerrado, Formações Pioneiras, Formações Pioneiras (Mangue) e Outros Usos (veja Governo de Sergipe, 2014 [19] para detalhamento da obtenção e classificação dos dados).

A avaliação dos remanescentes florestais de Umbaúba foi feita em uma escala de paisagem, na qual calculamos métricas de paisagem direcionadas aos fragmentos e as classes (i.e., conjunto de fragmentos do mesmo tipo vegetacional) existentes no município. As métricas calculadas incluem a área e o perímetro das manchas, o índice de circularidade (RCC) que mede o nível de irregularidade no formato que o fragmento assume, além de controlar o efeito da área do fragmento, a contiguidade (CONTI) que mede o grau de continuidade dos fragmentos em relação a toda paisagem, descrevendo o efeito de perda e fragmentação de habitat; a proporção da classe relativa à paisagem e o índice da maior mancha, que descrevem respectivamente a porcentagem que a classe e o maior fragmento ocupam na paisagem, e por fim, o índice de proximidade (PROX) que considera o tamanho e a distância de uma mancha focal, em relação a todas as outras da mesma classe dentro de um raio arbitrariamente pré-determinado (1500 metros neste caso) (Tabela 1). Os valores obtidos estão baseados na regra de vizinhança dos 8 vizinhos, a qual considera conexões laterais e diagonais. As análises foram executadas com o auxílio do software Fragstats versão 4.2 [21]. 


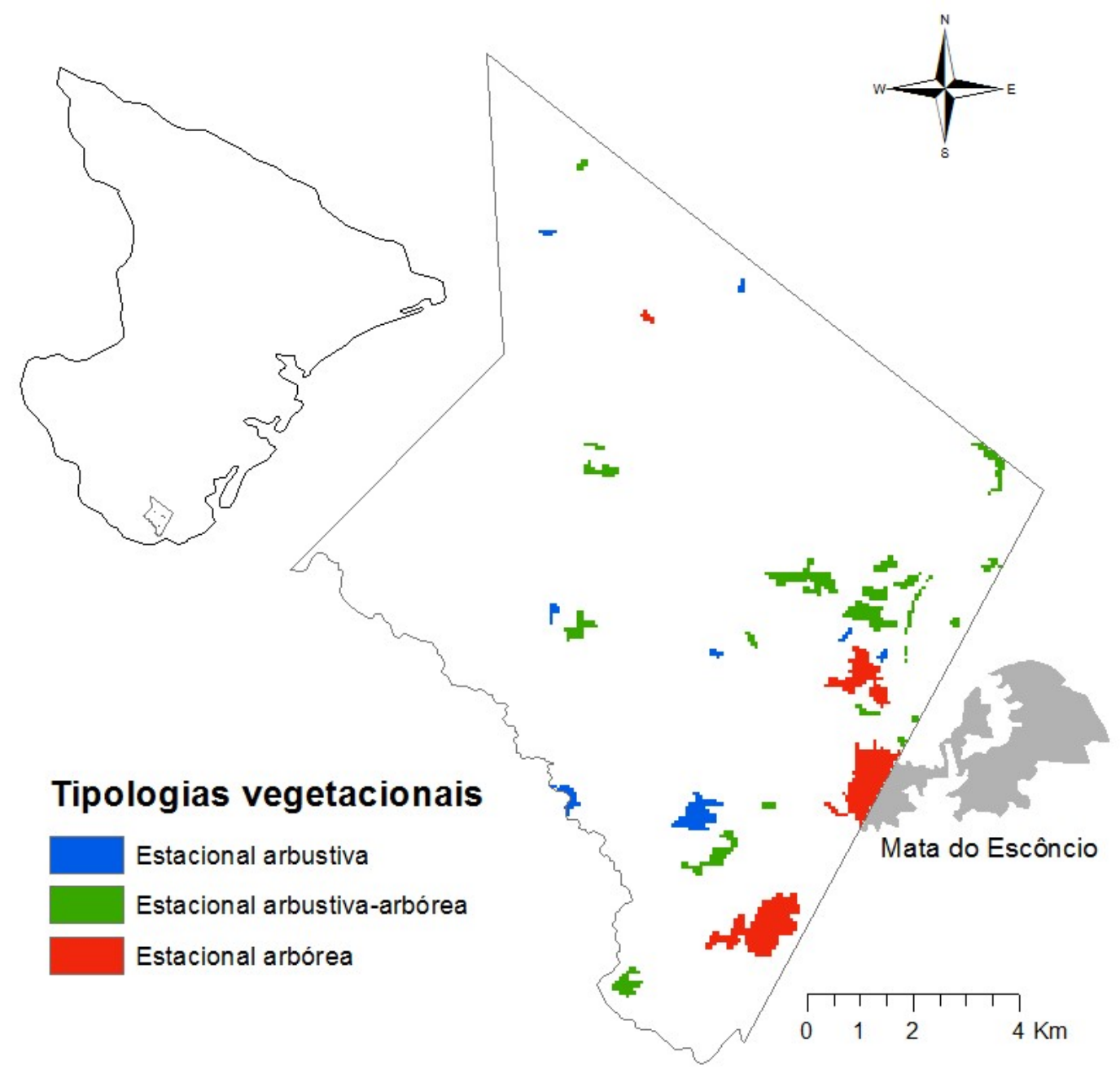

Figura 1- Localização do município de Umbaúba, mostrando a classificação dos remanescentes florestais e destacando a Mata do Escôncio (veja texto principal). 
Tabela 1- Descrição das métricas de fragmento e de classes de paisagem utilizadas para caracterizar o padrão de cobertura florestal do município de Umbaúba, Sergipe.

\begin{tabular}{|c|c|c|}
\hline MÉTRICA & FÓRMULA & DESCRIÇÃO \\
\hline Área & - & Tamanho da mancha (ha) \\
\hline Perímetro & & Perímetro da mancha (em m) \\
\hline $\begin{array}{l}\text { Índice de Circularidade } \\
(R C C)\end{array}$ & {$\left[1-\frac{C_{i}}{c_{i}}\right]$} & $\begin{array}{l}C_{i}=\text { área em } \mathrm{m}^{2} \text { da mancha } i, \mathrm{c}_{\mathrm{i}}=\text { área em } \\
\mathrm{m}^{2} \text { do menor círculo circunscrito ao redor } \\
\text { da mancha } i .0<\mathrm{RCC}<1 \text {, onde } 1 \text { indica } \\
\text { irregularidade máxima. }\end{array}$ \\
\hline Contiguidade (CONTI) & $\left\lceil\frac{\sum_{r=1}^{z} b_{i r}}{a_{i}}-1\right.$ & $\begin{array}{l}b_{i r}=\text { valor de contiguidade para o pixel } r \text { na } \\
\text { mancha } \mathrm{i} \\
v=\text { soma dos valores em células } 3 \times 3 \\
a_{i}=\text { área da mancha } i \text { em número de } \\
\text { células. Tende a zero quando o fragmento } \\
\text { for formado por apenas } 1 \text { pixel e aumenta } \\
\text { até } 1 \text { para um fragmento que ocupa toda } \\
\text { paisagem. }\end{array}$ \\
\hline $\begin{array}{l}\text { Porcentagem da classe } \\
\text { relativo à paisagem }\end{array}$ & $\sum \mathrm{a}_{\mathrm{i}} / \mathrm{A} \frac{\sum_{j=1}^{n} \square}{A} \times 100$ & $\begin{array}{l}a_{i}=\text { área em } \mathrm{m}^{2} \text { da mancha } i . \\
A=\text { área da paisagem em }\left(\mathrm{m}^{2}\right) .\end{array}$ \\
\hline $\begin{array}{l}\text { Índice da maior } \\
\text { mancha }\end{array}$ & $\frac{\max \left(a_{i}\right)}{A} \times 100$ & $\begin{array}{l}a_{i}=\text { área em } \mathrm{m}^{2} \text { da mancha } i \\
\text { Porcentagem da maior mancha de uma } \\
\text { classe relativo à paisagem, onde } A=\text { área } \\
\text { da paisagem em }\left(\mathrm{m}^{2}\right) .\end{array}$ \\
\hline $\begin{array}{l}\text { Índice de proximidade } \\
(\mathrm{PROX})\end{array}$ & $\sum_{s=1}^{n} \frac{a_{i j}}{h_{i j}^{2}}$ & $\begin{array}{l}a_{i j}=\text { área em } \mathrm{m}^{2} \text { da mancha } \mathrm{j} \text { dentro do raio } \\
\text { especificado para a mancha } i \text {. } \\
h_{i j}=\text { distância entre a mancha } i \text { e a mancha } j \\
\text { baseado na distância borda-borda. Tende a } \\
\text { zero quando o fragmento está isolado na } \\
\text { paisagem e aumenta com a proximidade de } \\
\text { manchas da mesma classe. }\end{array}$ \\
\hline
\end{tabular}

\section{RESULTADOS E DISCUSSÃO}

O município contém 35 fragmentos florestais de três classes distintas de floresta estacional: arbustiva, arbustivo-arbórea e arbórea, os quais correspondem a um total de 503 ha. A tipologia vegetacional estacional arbórea apresentou a maior cobertura (235 ha; 46,7\%) e o menor número de manchas, apenas quatro $(11,4 \%)$. Por outro lado, apesar do tipo estacional arbustivoarbóreo cobrir a segunda maior área da paisagem, o mesmo possui um elevado número de manchas (23 fragmentos) refletindo o alto nível de fragmentação desta classe (Figura 2A).

As medidas de forma foram semelhantes entre as classes de vegetação (Figura 2B) (estacional arbustiva $=0,62$, estacional arbustiva-arbórea $=0,61$, estacional arbórea $=0,64$ ). Isso indica que os remanescentes florestais de Umbaúba possuem formatos irregulares, ou seja, que desviam da forma circular, a qual é considerada ideal para conservação. Esse padrão implica em um maior efeito de borda dos remanescentes, independentemente de sua área. Os efeitos específicos da maior exposição do fragmento à matriz para este caso em particular não são 
conhecidos, especialmente em um contexto de escassez de informações sobre a biota local e suas interações. Entretanto, de modo geral, espera-se que esse processo envolva 1) mudanças microclimáticas resultantes da maior exposição do remanescente às condições da matriz circundante; 2) mudanças nos padrões de abundância e distribuição de espécies resultante da alteração das condições locais; e consequentemente 3) alterações na estrutura da comunidade local, incluindo o favorecimento de espécies mais tolerantes e generalistas [22].

Com relação contiguidade, os fragmentos de floresta estacional arbórea mostraram os maiores valores $(0,734 \mathrm{em}$ uma escala onde o valor máximo é 1$)$, indicando que a paisagem apresenta menor grau de perda de habitat e fragmentação dos remanescentes nesta classe vegetacional (Figura 2B). Embora os valores totais sejam criticamente baixos, esse resultado indica um potencial para medidas de conservação que envolvam restabelecer conexões entre os remanescentes, sobretudo pelo fato de estes apresentarem uma estrutura vegetal em estágio sucessional mais avançado e ter o potencial para abrigar maior diversidade de espécies, incluindo espécies menos tolerantes a perturbações ambientais [23].

Além da formação estacional arbórea apresentar os melhores resultados para a maioria das métricas calculadas, o fato de Umbaúba ser um grande consumidor de produtos energéticos florestais reforça a indicação desta fisionomia como prioritária para medidas de conservação dentro do município [19]. Neste contexto, merece destaque o fragmento conhecido como Mata do Escôncio ( $\left.37^{\circ} 37^{\prime} \mathrm{W}, 11^{\circ} 26^{\prime} \mathrm{S}\right)$, o qual possui uma área comparativamente grande no município de Umbaúba (segundo maior fragmento) e estendendo-se ao município vizinho de Indiaroba, formando um remanescente de 544 ha. Além de manter populações do macaco-guigó (Callicebus coimbrai), espécie ameaçada de extinção [24], este fragmento abriga também a nascente do rio Indiaroba, um relevante componente socioambiental, com importância para a manutenção de serviços ecossistêmicos locais, indicando uma em potencial para integrar medidas locais de conservação. As outras tipologias vegetacionais, por outro lado, em função dos seus baixos valores de cobertura e a descontinuidade (Figura 2C), ressaltam o alto grau de perda de área e de diversidade nas florestas do município, sugerindo a necessidade de medidas urgentes de recuperação florestal. De fato, devido a elevada perda e fragmentação de habitat no município, entre as medidas mais pertinentes consistem, sobretudo, em práticas de restauração, recuperação e reconexão dos remanescentes, incluindo a implantação de corredores florestais, manejo da matriz ou aumento das áreas florestais, resultando em uma maior conectividade entre os remanescentes e facilitando o movimento e estabelecimentos de espécies no local [23].

Uma gama de métricas de paisagens tem sido elaborada, as quais podem descrever inúmeros aspectos da composição, estrutura, distribuição e configuração espacial de habitats em escala de paisagem [4]. Atrelado a isso está o desenvolvimento e disponibilização de plataformas computacionais que permitem o processamento e análise destas informações [25]. O uso dessas ferramentas já é bem consolidado em estudos de ecologia envolvendo diversos aspectos do padrão e da dinâmica espacial de processos ecológicos em distintos níveis de organização [26, 27]. Além disso, quando dados biológicos são escassos, estas ferramentas podem ser uma alternativa simples e eficaz para a orientação na tomada de decisões de conservação em escala local (e.g., municipal), ou mesmo como uma etapa preliminar da caracterização das condições ambientais locais [3, 28]. No caso do município de Umbaúba, a lacuna no conhecimento biológico local é um fator limitante para se definir prioridades locais de medidas de conservação. No entanto, a utilização de métricas e elementos de paisagens, juntamente com a vinculação de outras informações ambientais locais, podem fornecer um primeiro subsídio para a tomada de decisões de conservação e recuperação dos recursos florestais no município, bem como permitir o direcionamento de novos estudos envolvendo as respostas da biota local à atual configuração da paisagem nessa região [29].

Um fator crítico, entretanto, para a análise e interpretação de padrões espaciais de configuração de habitats é escala espacial adotada [30] A estratégia de restringir a avaliação das métricas de paisagem em escala municipal está pautada no Plano Municipal de Conservação e Recuperação da Mata Atlântica (PMMA) que objetiva definir as diretrizes de conservação e manejo da Mata Atlântica em cada município, através de suas prefeituras e órgãos ambientais, ao longo do domínio do bioma no Brasil. Apesar de ser uma iniciativa pertinente e necessária, sobretudo no que trata de promover um maior envolvimento dos atores locais na conservação 
dos seus remanescentes, a abordagem por município pode limitar as análises e consequentemente a tomada de decisões em outras escalas administrativas. O município estudado aqui, Indiaroba, ilustra essa problemática, uma vez que seu remanescente com indicadores de paisagem mais favoráveis (a mata do Escôncio) excede o limite municipal, demandando, portanto, uma atuação em outros níveis administrativos para a intervenções de conservação para o remanescente como um todo. Portanto, para uma agenda articulada entre diferentes setores da sociedade, é fundamental que essas estratégias estejam alinhadas às análises em outras escalas espaciais e administrativas, como a Estadual. Aliado a isso, outra ferramenta importante para a conservação é o Zoneamento Ecológico Econômico (ZEE), que definirá e ordenará o espaço para compatibilizar diferentes usos do solo, como o crescimento urbano, desenvolvimento agrícola e conservação de biodiversidade. Em Sergipe, o ZEE ainda está em fase de planejamento. É crucial, portanto, que esse instrumento de gestão ambiental seja subsidiado pela maior quantidade de informações possível sobre as condições ambientais do estado, incluindo a configuração dos remanescentes florestais, de modo a promover um melhor suporte nas tomadas de decisão e permitir a compatibilização das atividades humanas com a proteção dos recursos naturais e serviços ecossistêmicos [31]. 
A

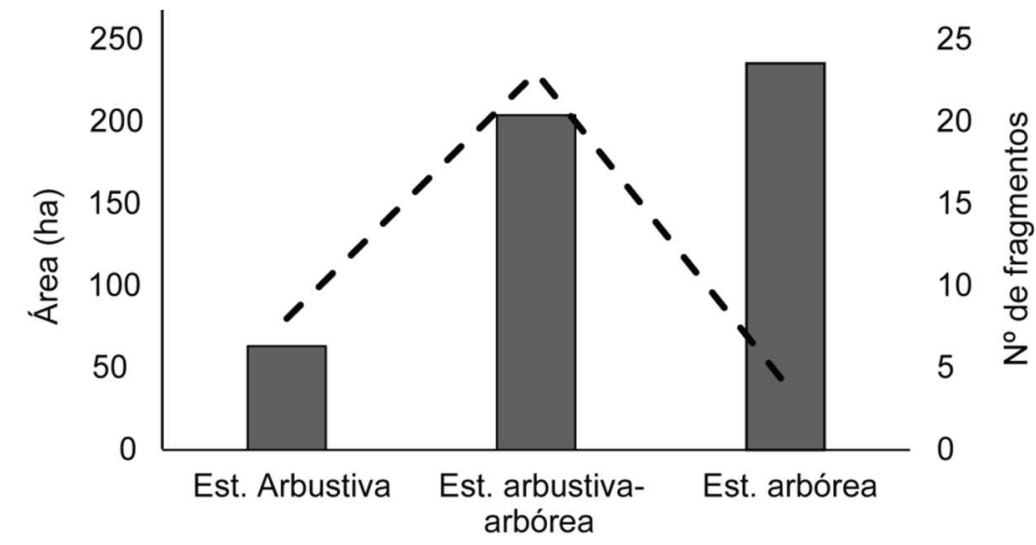

Tipologia vegetacional

$\square$ Área $-\mathrm{N}^{\circ}$ de fragmentos

B

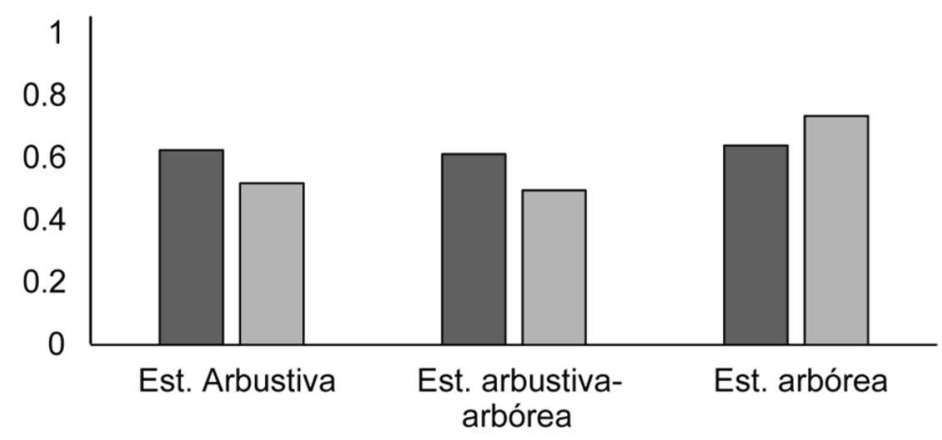

$\square \mathrm{RCC}$

Tipologia Vegetacional

$\square$ CONTI

C

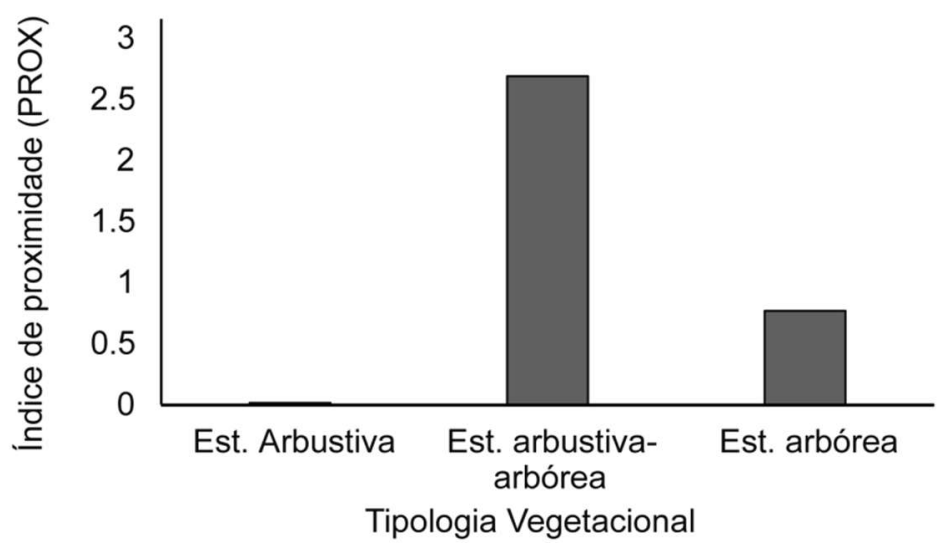

Figura 2 - Medidas das métricas calculadas para as tipologias vegetacionais. " $A$ " mostra a relação do número de fragmentos e a área de cobertura de cada classe na paisagem; " $B$ " mostra medidas de forma (Índice de Circularidade (RCC) e Contiguidade (CONTI)) para cada classe e "C" medida de agregação mostrando o nível de proximidade entre os fragmentos da mesma classe na paisagem. Est. = Estacional. 


\section{CONCLUSÃO}

A situação da Mata atlântica no município de Umbaúba, Sergipe, apresenta elevados indicadores de degradação florestal, tanto pela baixa quantidade quanto pela qualidade dos fragmentos. Enquanto a formação estacional arbustiva-arbórea apresentou uma configuração espacial propicia para ações de recuperação florestal, os remanescentes de florestas estacionais arbóreas do município incluem áreas relevantes para a conservação, incluindo espécies em perigo de extinção e serviços ecossistêmicos relevantes. Diante da demanda por produtos energéticos de origem florestal no município, é fundamental garantir a proteção legal dos remanescentes nativos de Mata Atlântica, compatibilizando-a com outros usos da terra que garantam o abastecimento energético e de outros serviços ambientais para a região.

\section{AGRADECIMENTOS}

Este estudo foi produzido durante a disciplina Ecologia da Paisagem (Turma 02, 2015.1) do Bacharelado em Ecologia da Universidade Federal de Sergipe. Agradecemos à Profa. Adriana Bocchiglieri pelas sugestões em uma versão preliminar deste artigo, à Universidade Federal de Sergipe pelas bolsas de IC a ERN e JLS, e ao CNPq pelo apoio a SFG (Proc. 476528/2013-5).

\section{REFERÊNCIAS BIBLIOGRÁFICAS}

1. Fischer J, Lindenmayer, DB. Landscape modification and habitat fragmentation: a synthesis. Global Ecology and Biogeography. 2007;16:265-280, doi: 10.1111/j.1466-8238.2006.00287.x.

2. Gardner TA, Barlow J, Chazdon R, Ewers RM, Harvey CA, Peres CA, Sodhi NS. Prospects for tropical forest biodiversity in a human-modified world. Ecology Letters. 2009;12:561-582, doi: 10.1111/j.1461-0248.2009.01294.x.

3. Metzger JP. Como lidar com regras pouco óbvias para conservação da biodiversidade em paisagens fragmentadas. Natureza \& Conservação. 2006;Out 4(2):11-23.

4. Uuemaa E, Antrop M, Roosaare M, Marja R, Mander U. Landscape metrics and indices: an overview of their use in landscape research. Liv. Rev. Landscape Research. 2009;3:1-28.

5. Forman RTT. Some general principles of landscape and regional ecology. Landscape Ecology. 1995; 10:133:142.

6. Fahrig L. Effects of habitat fragmentation on biodiversity. Annual Reviews in Ecology \& Systematics. 2003 3: 487-515. doi: 10.1146/annurev.ecolsys.34.011802.132419

7. Ranta P, Blon T, Niemelã J, Joensuu E, Siitonen M. The fragmented Atlantic rain forest of Brasil: size, shape and distribution of Forest fragments. Biodiversity and Conservation. 1998 7: 385-403.

8. Metzger JP. Estrutura da paisagem: o uso adequado de métricas. In: Cullen Junior, Pádua L, C.V.\& Rudran, R. Métodos de estudos em biologia da conservação e manejo da vida Silvestre. Curitiba: Ed. UFPR/ Fundação O Boticário de Proteção à natureza; 2003.p.539-553.667p.

9. Ribeiro MC. Metzger JP, Martensen AC, Ponzoni FJ, Hirota MM. The Brazilian Atlantic Forest: How much is left, and how is the remaining forest distributed? Implications for conservation. Biological Conservation. 2009;142;1141-1153, doi:10.1016/j.biocon.2009.02.021.

10. Rizzini CT. Tratado de Fitogeografia do Brasil: aspectos ecológicos, sociológicos e florísticos. Âmbito Cultural Edições Ltda., Rio de Janeiro. 1997. 747p.

11. Ab'saber AN. OS Domínios da Natureza no Brasil; potencialidades paisagísticas. São Paulo: Ateliê Editorial, 2003. 151p.

12. Myers N, Mittermeier RA, Mittermeier CG., da Fonseca GAB \& Kent J. Biodiversity hotspots for conservation priorities. Nature. 2000;403:853-858.

13. Pinto LP, Brito CW. Dinâmica da perda da biodiversidade na Mata Atlântica brasileira: uma introdução. SOS Mata Atlântica/ Conservação Internacional do Brasil. Belo Horizonte. 2005 27-30.

14. Coimbra FAF, Câmara IBG. Os limites originais do Bioma Mata Atlântica na Região Nordeste do Brasil. São Paulo: Fundação Brasileira para a Conservação da Natureza. 1996. 86p. 
15. Gascon C, Laurance WF, Lovejoy TE. Fragmentação florestal e biodiversidade na Amazônia central. IN: Conservação da biodiversidade em ecossistemas tropicais, Garay, I \& Dias, B. (eds.), Editora Vozes. 2000;174-189.

16. Fundação SOS Mata Atlântica, 2015. www.fundacaososmataatlantica.org.br. Acesso em 26 de novembro de 2015.

17. Fundação SOS Mata Atlântica. Atlas dos remanescentes florestais da Mata Atlântica, 2014.

18. Siqueira ER, Ribeiro FE. Recursos genéticos vegetais da mata atlântica de Sergipe. In:Mata Atlântica de Sergipe. Aracaju: EMBRAPA/ CPATC; 2001. p. 51-76.

19. Governo de Sergipe, Diagnóstico Florestal de Sergipe 2014. / Secretária de Estado de Meio Ambiente e dos Recursos Hídricos. Aracaju: [s.n.], 2014.

20. IBGE - Instituto Brasileiro de Geográfica e Estatística, 2007. www.ibge.br. Acesso em 26 de novembro de 2015.

21. McGarigal K, Ene. FRAGSTATS v4: Spatial Pattern Analysis Program for Categorical and Continuous Maps. Computer software program produced by the authors at the University of Massachusetts, Amherst. 2012.

22. Murcia, C. Edge effects in fragmented forests: implications for conservation. Trends in Ecology and Evolution. 1995;10:58-62.

23. Rocha CFD, Bergallo HG, Sluys MV, Alves MAS, Jenkins C. Biologia da conservação: Essências. São Carlos: RiMa; 2006, Capítulo 13, Corredores ecológicos e conservação da biodiversidade: Um estudo de caso da Mata Atlântica; p. 317-342.

24. Jeruzalinsk L, Oliveira MM, Pereira RF, Santana V, Bastos PCR, Ferrari SF. Preliminary Evaluation of the Conservation Status of Callicebus coimbrai Kobayashi \& Langguth, 1999 in the Brazilian State of Sergipe. Primate Conservation. 2006;21:25-32.

25. Turner MG. Landscape Ecology: what is the state of the science? Annu. Rev. Ecol. Evol. Syst. 2005; Aug 36:314-344, doi: 10.1146/annurev.ecolsys.36.102003.1.

26. Herzog F, Lausch A, Müller E, Thulke HH, Steinhardt U, Lehmann S. Landscape Metrics for Assessment of Landscape Destruction and Rehabilitation. Environmental Management. 2001; 27(1): 91-107, doi: 10.1007/s002670010136.

27. Millington AC, Velez-Linedo XM, Bradley AV. Scale dependence in multitemporal mapping of forest fragmentation in Bolivia: implications for explaining temporal trends in landscape ecology and applications to biodiversity conservation. Journal of Photogrammetry \& Remote Sensing. 2003; Oct 57:289-299, doi: 10.1016/S0924-2716(02)00154-5.

28. Banks-Leite C, Ewers RM, Kapos V, Martensen AC, Metzger JP. Comparing species and measures of landscape structure as indicators of conservation importance. Journal of Applied Ecology. 2011; 48:706-714, doi: 10.1111/j.1365-2664.2011.01966.x

29. Cunha AA, Guedes FB. Mapeamentos para conservação e recuperação da biodiversidade na Mata Atlântica: em busca de uma estratégia espacial integradora para orientar ações aplicadas. Ministério do Meio Ambiente (MMA), Secretaria de Biodiversidade e Florestas. Brasília, DF; 2013. Capítulo 7, Perspectivas para a integração de dados e uso de sistemas de informação geográfica e da ecologia de paisagens para a tomada de decisão para a conservação e recuperação da biodiversidade da Mata Atlântica brasileira; p. 201-216.

30. Wu J, Qu Y. Dealing with scale in landscape analysis: An overview. Geographic Information Sciences 2000;6:1-5.

31. Cunha AA, Guedes FB. Mapeamentos para conservação e recuperação da biodiversidade na Mata Atlântica: em busca de uma estratégia espacial integradora para orientar ações aplicadas. Ministério do Meio Ambiente (MMA), Secretaria de Biodiversidade e Florestas. Brasília, DF; 2013. Espécies, ecossistemas, paisagens e serviços ambientais: uma estratégia espacial integradora para orientar os esforços de conservação e recuperação da biodiversidade na Mata Atlântica; p. 11-28. 